\title{
Neuropsychologische Prädiktoren für den Erfolg beruflicher Wiedereingliederung
}

Stefan Watzke ${ }^{1}$, Peter Brieger ${ }^{2}$

${ }^{1}$ Universitätsklinik für Psychiatrie, Psychotherapie und Psychosomatik der Martin-Luther-Universität Halle-Wittenberg; ${ }^{2}$ kbo-Isar-Amper-Klinikum, Haar bei München

\section{ZUSAMMENFASSUNG}

Kognitive Leistungseinschränkungen stellen zentrale Erkrankungsmerkmale der Schizophrenie und wichtige Prognosefaktoren für den funktionalen Outcome Betroffener dar. In der beruflichen Rehabilitation werden sie daher als Zielgrößen für Trainings einerseits und als Erfolgsprädiktoren andererseits genutzt. Bei zunehmender Diversifizierung der rehabilitativen Angebote und der angesprochenen Klientel stellt sich die Frage, ob kognitive Defizite zielgruppen- und maßnahmenübergreifend ihre wichtige Funktion beibehalten können.

Eine Durchsicht der Literatur legt nahe, dass die zentralen Aussagen zu prognostischer Validität und Adressierbarkeit mittels kognitiver Trainings auf Patienten mit affektiven Störungen grundsätzlich ausgeweitet werden kann. Für Rehabilitanden anderer diagnostischer Gruppen liegen kaum Studien zur Erfolgsprognose vor. Es muss aber davon ausgegangen werden, dass bei nur geringen kognitiven Beeinträchtigungen andere Merkmale - z. B. Angst, Metakognitionen und Fähigkeiten der sozialen Kognition - zur Prognoseabschätzung herangezogen werden müssen.

\section{Einleitung}

In einer früheren Arbeit [1] wendeten wir uns der Frage zu, wie sich der Erfolg beruflicher Rehabilitationsbemühungen für Patienten mit schweren psychischen Erkrankungen prognostizieren lässt. Prämisse dieser Studie war die Feststellung, dass psychische Störungen zwar mit einem weiten Spektrum erkrankungsbezogener Beeinträchtigungen verbunden sind, sich aber gerade diese primär klinischen Merkmale - aktuelle Symptomatik, Diagnose oder Verlauf - nur in geringem Umfang zur Vorhersage von Rehabilitationserfolg nutzen lassen [2-4]. Zeitgleich legten Green et al. [5] eine einflussreiche Arbeit vor, die zeigte, dass - zumindest für Patienten mit Schizophrenie - die kognitiven Leistungen dieser Patienten zur Vorhersage des „Zurechtkommens im Alltag“ bevorzugt genutzt werden kann.

Wir adaptierten diese Überlegung und suchten für Patienten mit Schizophrenie nach Studien, die explizit den Erfolg beruflicher Rehabilitation mit kognitiven Leistungsmaßen in Zusammenhang brachten [1]. Der damalige Überblick zeigte, dass $20-45 \%$ des individuellen Rehabilitationserfolges durch kognitive Leistungen erklärt wird.

Einiges hat sich seither in verschiedenen Aspekten des Arbeitsfeldes verändert. Während berufliche Rehabilitationsprogramme für Menschen mit schweren psychischen Erkrankungen ursprünglich in erster Linie auf die Beeinträchtigungen von Patienten mit Schizophrenie zugeschnitten waren $[3,6]$, findet sich heute ein umfassenderes diagnostisches Spektrum psychischer Störungen unter den Teilnehmern der Rehabilitationsprogramme. So waren in einem breit evaluierten RPK-Programm [7] zwar ca. ein Viertel der Rehabilitanden an psychotischen Störungen erkrankt, ein weiteres Viertel litt aber unter depressiven Störungen. Persönlichkeitsstörungen waren als Primärdiagnose mit $17 \%$ vertreten, gefolgt von Patienten mit Angst- und Zwangserkrankungen (9\%), Belastungsstörungen (9\%) und somatoformen Störungen (8\%). Diese Veränderung ist dem Umstand geschuldet, dass sich die Zahl erkrankungsbedingter Arbeitsunfähigkeitstage [8], rehabilitativer Leistungen sowie Erwerbsminderungsrenten [9] in den letzten Jahren erhöht hat und in den meisten Fällen ursächlicher psychischer Erkrankungen auf depressive Episoden, Reaktionen auf schwere Belastungen und Anpassungsstörungen sowie auf „andere neurotische Störungen“ zurückgehen [10]. Während diese Zahlen primär aus Publikationen der Kostenträger stammen, finden sich national und international publizierte Studien zur Prognose des Erfolges rehabilitativer Maßnahmen in weit geringerem Umfang für diese veränderten Zielgruppen.

Auf Seite der Angebote zeigt sich eine Veränderung der Rehabilitationsprogramme von klassischen Maßnahmen des Train-and-Place hin zu Konzepten des Place-and-Train, wie sie durch Programme des „Supported Employment“ oder Modelle des „Individual Placement and Support“ [11-13] umgesetzt werden. Die Frage nach der Übertragbarkeit der Befunde zu kognitiven Leistungen als valide Prädiktoren des Rehabilitationserfolges in einer sich verändernden Landschaft rehabilitativer Angebote ist bedeutsam, wurden die Programme des Supported Employment explizit für „individuals with the most significant disabilities “ [11; S. 222] konzipiert und kompensieren alle Einschränkun- 
gen, die der Betroffene aufweist. Hier verändert sich die Frage nach Prädiktoren des Wiedereingliederungserfolges hin zur Notwendigkeit, zu kompensierende Beeinträchtigungen festzustellen und Hilfen nach ihnen auszurichten.

In beiden Fällen stellt sich jedoch die Frage, ob kognitive Leistungseinschränkungen bei den zur Diskussion stehenden Erkrankungen in substantiellem Ausmaß vorliegen - einerseits um sie einer Nutzung als Erfolgsprädiktoren zugänglich zu machen, andererseits um geeignete Kompensationen für gegebenenfalls vorliegende Defizite zu entwickeln. Dieser Frage wird im folgenden Abschnitt überblicksartig nachgegangen.

Um dieser Frage systematisch nachzugehen, wurde eine PubMed-Literatursuche mit folgenden Schlüsselbegriffen gestartet: (cognitive or cognition or attention or memory or executive function) and (functional outcome or work outcome or employment or vocational rehabilitation or work rehabilitation) and (mental illness or schizophrenia or affective disorder or depression or bipolar disorder or personality disorder or anxiety). Der folgende Abschnitt fasst das Ergebnis dieser Suche überblicksartig zusammen.

\section{Kognitive Einschränkungen, funktionaler Outcome und Rehabilitationserfolg bei psychischen Störungen}

\section{Schizophrenie}

Dass sich Schizophrenie als neurokognitive Störung verstehen lässt $[14,15]$, gilt heute als gesichert. Kognitive Defizite werden als zentrale und überdauernde Merkmale der Erkrankung angenommen [16] und als zum Erscheinungsbild der Schizophrenie gehörend postuliert wie die positiven und negativen Symptome selbst [17]. Kognitive Störungen lassen sich sowohl bei ersterkrankten Personen [18], Patienten mit florider Symptomatik als auch in symptomatischer Remission [19, 20] nachweisen. Als bei Schizophrenie besonders beeinträchtigte Domänen kognitiver Leistungen wurden die Verarbeitungsgeschwindigkeit, Vigilanz, Arbeitsgedächtnis, das sekundär verbale Gedächtnis, Wortflüssigkeit, psychomotorischen Fertigkeiten und die Exekutivfunktionen identifiziert, die zudem geeignet sind, soziales Outcome (soziale Netzwerke, unabhängige Lebensführung, berufliches Fortkommen) zu prognostizieren $[1,5,21,22]$.

Bei über 1000 Personen mit schweren psychischen Erkrankungen vergleichen Metcalfe et al. [23] strukturelle, individuell biografische und kognitive Prädiktoren einer erfolgreichen Wiedereingliederung durch Programme des Individual Placement and Support. Neben der individuellen Arbeitsbiographie und der Dauer der Beschäftigungslosigkeit vor der Maßnahme erwiesen sich kognitive Leistungen als valide Vorhersagegrößen. Auch in anderen Studien fin- den sich die Verarbeitungsgeschwindigkeit als basales Maß [24], Aufmerksamkeitsleistung, verbales Gedächtnis und Exekutivfunktionen [25-27] als Prädiktoren eines Wiedereingliederungserfolges schizophrener Patienten. In einer Studie von Puig et al. [28] erwiesen sich im Rahmen eines Trainings verbesserte Aufmerksamkeitsleistungen als Prädiktoren einer längeren Verweildauer in Beschäftigung.

\section{Affektive Störungen}

Auch wenn Patienten mit bipolar affektiven Störungen in Programmen zur beruflichen Rehabilitation unterrepräsentiert scheinen, liegen doch gesicherte Befunde zu kognitiven Beeinträchtigungen bei diesen Erkrankungen vor [29]. Auch hier treten Defizite in den Exekutivfunktionen, dem verbalen Gedächtnis, im visuell-räumlichen Gedächtnis sowie in der Aufmerksamkeit auf. Diese stehen einerseits mit der jeweiligen Psychopathologie in Zusammenhang [30] - anderseits persistieren diese z. T. in euthyme Erkrankungsphasen [31]. Martinez-Aran et al. [32] verglichen die kognitiven Leistungen bei bipolar affektiven Patienten in manischen, depressiven und euthymen Erkrankungsphasen und fanden kognitive Defizite in allen Gruppen in frontal-exekutiven Funktionen, in Aufmerksamkeitsleistungen sowie im verbalen Lernen und Gedächtnis. Im logischen Gedächtnis fanden sich Defizite nur in akut-manischen Erkrankungsphasen, im nonverbalen Gedächtnis erwiesen sich vorrangig Patienten in depressiven Episoden beeinträchtigt. Insgesamt sind kognitive Beeinträchtigungen bei bipolar affektiven Erkrankungen im Vergleich zu Patienten mit Schizophrenie aber als milder und weniger generalisiert zu beschreiben [33, 34], stehen aber dennoch mit funktionalem Outcome in engem Zusammenhang [35, 36].

Für unipolar depressive Störungen zeigen Metaanalysen $[37,38]$ Beeinträchtigungen ebenfalls in Verarbeitungsgeschwindigkeit, Aufmerksamkeit, Gedächtnis und exekutiven Kontrollfunktionen. Zumindest für Exekutivfunktionen und Aufmerksamkeit fanden sich persistierende Störungen auch über depressive Phasen hinaus, für Gedächtnisstörungen ließ sich dieser Effekt nur tendenziell abbilden [37, 39]. In einem systematischen Review [40] folgern Woo et al. dennoch, dass kognitive Beeinträchtigungen bei Patienten mit unipolar affektiven Störungen als Kernmerkmal der Erkrankung angesehen werden sollen. Diese seien nicht als Epiphänomene zu bewerten, die ausschließlich Folge der affektiven Symptomatik sind und mit deren An- bzw. Abwesenheit fluktuieren.

Für Patienten mit uni- und bipolar affektiven Störungen liegen Prognosestudien zum Rehabilitationserfolg nicht explizit vor [40], obwohl Schwierigkeiten in beruflichen Funktionsbereichen zu den häufigsten sozialen Folgen depressiver Störungen zählen [41]. Boland et al. [42] berichten aber, dass Patienten mit bipolarer Störung dann länger ohne Beschäftigung waren und häufiger ihre Anstellung 
verloren, wenn sie über schlechtere generelle kognitive Leistungen verfügten.

Woo und Kollegen [40] tragen Studien für Patienten mit Depression zusammen und postulieren, dass sich Defizite in Aufmerksamkeit, Lernfähigkeit, Gedächtnis und Exekutivfunktionen als Einflussgrößen schlechterer beruflicher Integration erweisen. Jaeger et al. [43] fanden, dass sich unabhängige Lebensführung depressiver Patienten (inklusive Arbeitsfähigkeit) durch kognitive Beeinträchtigungen prognostizieren ließ. Eine größere epidemiologische Studie [44] zeigte, dass persistierende Störungen in Aufmerksamkeit und Konzentration als Mediatoren zwischen Depressivität und eingeschränkter beruflicher Rollenerfüllung wirkten.

\section{Persönlichkeitsstörungen und „neurotische“ Erkrankungen}

Patienten mit Persönlichkeitsstörungen - insbesondere mit emotional instabiler Persönlichkeitsstörung - weisen geringgradige, vergleichsweise unspezifische und zeitlich instabile Beeinträchtigungen von Aufmerksamkeit, Gedächtnis und exekutiven Funktionen auf [45, 46]. Entsprechend finden sich Assoziationsstudien zwischen kognitiver Leistung und Funktionsniveau kaum, obwohl Persönlichkeitsstörungen mit schweren Beeinträchtigungen im beruflichen Funktionsniveau assoziiert sind [47, 48]. Bei Juurlink et al. [49] zeigte sich lediglich, dass die Arbeitsfähigkeit von Patienten mit Borderline-Persönlichkeitsstörung durch deren Bildung und die Anzahl spezifischer Störungssymptome vorhergesagt werden konnte.

Angststörungen scheinen nur wenig mit substantiellen kognitiven Beeinträchtigungen assoziiert zu sein. Lediglich eine erhöhte Aufmerksamkeit für bedrohliche Reize und in deren Folge veränderte Speicherprozesse werden beschrieben [50, 51]. Studien zum Zusammenhang kognitiver Leistungen und beruflicher Wiedereingliederung finden sich nicht. Muschalla [52] betont sogar, dass sich in einer Stichprobe von 1570 Personen mit Depressionen, Angst- und Anpassungsstörungen die Entlassprognose hinsichtlich einer beruflichen Perspektive nach psychotherapeutischer Behandlung nicht durch die kognitive Leistung der Patienten vorhersagen ließ. Wohl aber erwiesen sich die Angst vor einem Arbeitsantritt, negative Einstellungen gegenüber einer Berufstätigkeit und lange Arbeitsabwesenheit vor der Behandlung als prognostisch valide.

\section{Resümee und Ausblick}

In den Industrienationen zeigt sich seit einigen Jahren ein Trend zunehmend höherer geistiger Arbeitsanforderungen im allgemeinen Arbeitsmarkt [53], die in enger Wechselwirkung mit den kognitiven Leistungen der Arbeitnehmer stehen [54].
Kognitive Beeinträchtigungen limitieren berufliches Fortkommen psychisch Erkrankter ebenso wie den Erfolg beruflicher Wiedereingliederungsbemühungen. Damit ist dem Fazit unserer Arbeit aus 2004 zunächst nichts hinzuzufügen. Allerdings sind von schwerwiegenden kognitiven Funktionseinschränkungen, die zudem in Phasen symptomatischer Stabilität persistieren, primär Patienten mit Schizophrenie betroffen. Green und Kollegen kommen daher in einer Revision ihres Papers aus dem Jahr 2000 [5] zu dem Schluss, dass sich kognitive Defizite in dieser Patientengruppe besser zur Prognose beruflicher Funktionsfähigkeit eignen als zur Vorhersage primär sozialer Fertigkeiten [55].

Für Patienten mit affektiven Störungen gilt der Zusammenhang zwischen kognitiver Einschränkung und beruflicher Wiedereingliederung inhaltlich ebenso - bei geringen ausgeprägten kognitiven Störungen und engerer Kopplung an die Psychopathologie vermutlich aber entsprechend in weniger starkem Umfang.

Für Patientengruppen, die aktuell zwar substantiell in Maßnahmen beruflicher Rehabilitation auftreten, in deren psychopathologischer Phänomenologie aber kognitive Störungen in geringerem Maße relevant sind, liegen kaum Studien zur Thematik vor - hier ist anzunehmen, dass nicht kognitive Beeinträchtigungen die berufliche Wiedereingliederung erschweren, sondern andere Faktoren - wie z. B. berufsbezogene und soziale Ängste, negative Metakognitionen und Verhaltensstörungen [56].

Gemeinsam ist den Arbeiten zu verschiedenen Störungsgruppen jedoch, dass jeweils diejenigen Maße kognitiver Leistung im Vordergrund stehen, die sich bereits bei Green et al. [5, 14] als mit funktionalem Outcome bei Schizophrenie assoziiert erwiesen - nämlich Verarbeitungsgeschwindigkeit, Aufmerksamkeit, Arbeitsgedächtnis, sekundäres Gedächtnis und Exekutivfunktionen. Dies ist insofern nicht verwunderlich, als dass eine enge inhaltliche Beziehung zwischen kognitiver Leistung und beruflicher Funktionsfähigkeit einerseits augenscheinvalide, anderseits durch Studien belegt ist [40]. Probleme in Aufmerksamkeit, Arbeitsgedächtnis und im verbalen Lernen kollidieren mit dem Erwerb neuer arbeitsbezogener Fertigkeiten, Defizite in exekutiven Kontrollfunktionen erschweren die Planung, Ausführung und Regulation konkurrierender Arbeitsaufgaben. Umgekehrt verbessern in berufliche Rehabilitation integrierte kognitive Trainings die Erfolge der Programme deutlich [57-59].

Abschließend ist zu konstatieren, dass sich kognitive Prädiktoren in der beruflichen Wiedereingliederung von Menschen mit schweren psychischen Erkrankungen weiterhin mit hoher prognostischer Validität zur Vorhersage des Rehabilitationserfolges nutzen lassen. Zudem stellen kognitive Einschränkungen wichtige Zielgrößen für Trainings im Rahmen der Rehabilitation dar. Dies gilt in erster Linie für 
Patienten mit Schizophrenie, aber auch für Betroffene von uni- und bipolaren affektiven Störungen.

Für andere Teilnehmer - z. B. mit Persönlichkeitsstörungen oder Angsterkrankungen - stellen sich aktuell Maße sozialer Kognition als mess- und trainierbare Prognosevariablen verschiedenster Interventionen dar. Diese Fähigkeiten, Absichten und Pläne anderer Menschen wahrzunehmen, zu interpretieren und auf sie zu reagieren - kurz, soziale Interaktionen wirkungsvoll zu gestalten, scheinen einerseits in einem weiten Spektrum psychischer Störungen beeinträchtigt. Andererseits sind sie eng mit funktionalem Outcome psychischer Störungen assoziiert [60].

Interessenkonflikt

Es liegen keine Interessenkonflikte vor.

Korrespondenzadresse

Prof. Dr. Stefan Watzke

Universitätsklinik für Psychiatrie, Psychotherapie und Psychosomatik

der Martin-Luther-Universität Halle-Wittenberg

Julius-Kühn-Str. 7, 06112 Halle

Tel. 0345/5573542, Fax 0345/5573604

stefan.watzke@uk-halle.de

\section{Literatur}

[1] Watzke S, Brieger P. Neuropsychologische Diagnostik in der beruflichen Rehabilitation schizophrener Menschen. Fortschr Neurol Psychiatr 2004; 72: 643-651.

[2] Anthony WA, Jansen MA. Predicting the vocational capacity of the chronically mentally ill. Research and policy implications. Am Psychol 1984; 39: 537-544.

[3] Reker T. Arbeitsrehabilitation in der Psychiatrie. Darmstadt: Steinkopff 1998.

[4] Cook JA, Razzano L. Vocational rehabilitation for persons with schizophrenia. Recent research and implications for practice. Schizophr Bull 2000; 26: 87-103.

[5] Green MF, Kern RS, Braff DL et al. Neurocognitive deficits and functional outcome in schizophrenia. Are we measuring the „right stuff“? Schizophr Bull 2000; 26: 119-136.

[6] Aktion Psychisch Kranke. Bestandsaufnahme zur Rehabilitation psychisch Kranker. Zwischenbericht zum 31.03.2002. Bonn: Bundesministerium für Arbeit und Sozialordnung 2002.

[7] Brieger P, Watzke S, Galvao A, Hühne M, Gawlik B. Wie wirkt berufliche Rehabilitation und Integration psychisch kranker Menschen? Bonn: Psychiatrie-Verlag 2006.

[8] Marschall J, Hildebrandt S, Zich K, Tisch T, Sörensen J, Nolting HD, Burgart E, Woköck T. DAK-Gesundheitsreport 2018. Hamburg: DAK-Forschung 2018.
[9] Deutsche Rentenversicherung Bund (Hrsg.) Positionspapier der Deutschen Rentenversicherung zur Bedeutung psychischer Erkrankungen in der Rehabilitation und bei Erwerbsminderung. Berlin: Deutsche Rentenversicherung Bund 2014.

[10] Knieps F, Pfaff H, Hrsg. Gesundheit und Arbeit. Zahlen, Daten, Fakten. Bd. 2016. BKK Gesundheitsreport. Berlin: Medizinisch Wissenschaftliche Verlagsgesellschaft 2016.

[11] Bond GR, Becker DR, Drake RE et al. Implementing supported employment as an evidence-based practice. Psychiatr Serv 2001; 52: 313-322.

[12] Keller K, Kreß S, Kumbier-Jordan B, Theißing A, Hrsg. Medizinisch-berufliche Rehabilitation RPK und die Konzeption des Supported Employment. BAG-RPK 2015.

[13] Bond GR, Drake RE, Mueser KT et al. An update on supported employment for people with severe mental illness. Psychiatr Serv 1997; 48: 335-346.

[14] Green MF, Nuechterlein KH. Should schizophrenia be treated as a neurocognitive disorder? Schizophr Bull 1999; 25: 309-319.

[15] Tripathi A, Kar SK, Shukla R. Cognitive deficits in schizophrenia: Understanding the biological correlates and remediation strategies. Clin Psychopharmacol Neurosci 2018; 16: 7-17.

[16] Goldberg TE, Greenberg RD, Griffin S] et al. The effect of Clozapine on cognition and psychiatric symptoms in patients with schizophrenia. Br J Psychiatry 1993; 162: 43-48.

[17] Cleghorn JM. A neurodiagnostic approach to schizophrenia. Can J Psychiatry 1988; 33: 555-561.

[18] Bilder RM, Goldman RS, Robinson D et al. Neuropsychology of first-episode schizophrenia. Initial characterization and clinical correlates. Am J Psychiatry 2000; 157: 549-559.

[19] Gold JM, Green MF. Neurocognition in Schizophrenia. In: Kaplan HI, Sadock BJ (Hrsg). Kaplan and Sadock's Comprehensive Textbook of Psychiatry. Baltimore: Lippincott, Williams \& Wilkins; 2005: 1426-1448.

[20] Reed RA, Harrow M, Herbener ES et al. Executive function in schizophrenia. Is it linked to psychosis and poor life functioning? J Nerv Ment Dis 2002; 190: 725-732.

[21] Watzke S, Brieger P, Kuss $O$ et al. Learning potential and rehabilitation outcome in schizophrenia. A longitudinal study. Psychiatr Serv 2008; 59: 248-255.

[22] Watzke S, Brieger P, Wiedl KH. Prediction of vocational rehabilitation outcome in schizophrenia. Incremental prognostic validity of learning potential beyond basic cognitive performance. JCEP 2009; 8: 52-62.

[23] Metcalfe JD, Riley J, McGurk S et al. Comparing predictors of employment in Individual Placement and Support: A longitudinal analysis. Psychiatry Res 2018; 264: 85-90.

[24] Reddy LF, Kern RS. Supported employment among veterans with serious mental illness: the role of cognition and social cognition on work outcome. Schizophr Res Cogn 2014; 1 : 144-148.

[25] Kaneda Y, Jayathilak K, Meltzer H. Determinants of work outcome in neuroleptic-resistant schizophrenia and schizoaffective disorder: cognitive impairment and clozapine treatment. Psychiatry Res 2010; 178: 57-62.

[26] Tan B-L. Profile of cognitive problems in schizophrenia and implications for vocational functioning. Aust Occup Ther J 2009; 56: 220-228

[27] Chang WC, Man Tang JY, Ming Hui CL et al. Clinical and cognitive predictors of vocational outcome in first-episode schizophrenia: a prospective 3 year follow-up study. Psychiatry Res 2014; 220: 834-839. 
[28] Puig O, Thomas KR, Twamley EW. Age and improved attention predict work attainment in combined compensatory cognitive Ttaining and supported employment for people with severe mental illness. J Nerv Ment Dis 2016; 204: 869-872.

[29] King S, Stone JM, Cleare A et al. A systematic review on neuropsychological function in bipolar disorders type I and II and subthreshold bipolar disorders-something to think about. CNS Spectr 2019; 24: 127-143.

[30] Severus WE, Watzke S, Brieger P. Bipolare affektive Störungen. Teil II. Genetik, Kognition und Therapie. Fortschr Neurol Psychiatr 2008; 76: 294-308.

[31] Malhi GS, Ivanovski B, Hadzi-Pavlovic D et al. Neuropsychological deficits and functional impairment in bipolar depression, hypomania and euthymia. Bipolar Disord 2007; 9: 114-125.

[32] Martinez-Aran A, Vieta E, Reinares M et al. Cognitive function across manic or hypomanic, depressed, and euthymic states in bipolar disorder. Am J Psychiatry 2004; 161: 262-270.

[33] Altshuler LL, Ventura J, van Gorp WG et al. Neurocognitive function in clinically stable men with bipolar I disorder or schizophrenia and normal control subjects. Biol Psychiatry 2004; 56: 560-569.

[34] Bora E, Yucel M, Fornito A et al. Major psychoses with mixed psychotic and mood symptoms. Are mixed psychoses associated with different neurobiological markers? Acta Psychiatr Scand 2008; 118: 172-187.

[35] Green MF. Cognitive límpairment and functional outcome in schizophrenia and bipolar disorder. J Clin Psychiatry 2006; 67 suppl 9: 3-8; discussion 36-42.

[36] Dickerson F, Origoni A, Stallings C et al. Occupational status and social adjustment six months after hospitalization early in the course of bipolar disorder. A prospective study. Bipolar Disord 2010; 12: 10-20.

[37] Rock PL, Roiser JP, Riedel W] et al. Cognitive impairment in depression: a systematic review and meta-analysis. Psychol Med 2014; 44: 2029-2040.

[38] Snyder HR. Major depressive disorder is associated with broad impairments on neuropsychological measures of executive function: a meta-analysis and review. Psychol Bull 2013; 139: 81-132.

[39] Conradi H], Ormel J, Jonge P de. Presence of individual (residual) symptoms during depressive episodes and periods of remission: a 3-year prospective study. Psychol Med 2011; 41: 1165-1174.

[40] Woo YS, Rosenblat JD, Kakar R et al. Cognitive deficits as a mediator of poor occupational function in remitted major depressive disorder patients. Clin Psychopharmacol Neurosci 2016; 14: 1-16.

[41] Judd LL, Schettler PJ, Solomon DA et al. Psychosocial disability and work role function compared across the long-term course of bipolar I, bipolar II and unipolar major depressive disorders. J Affect Disord 2008; 108: 49-58.

[42] Boland EM, Stange JP, Molz Adams A et al. Associations between sleep disturbance, cognitive functioning and work disability in Bipolar Disorder. Psychiatry Res 2015; 230: 567-574.

[43] Jaeger J, Berns S, Uzelac S et al. Neurocognitive deficits and disability in major depressive disorder. Psychiatry Res 2006; 145: 39-48.

[44] Buist-Bouwman MA, Ormel J, Graaf R de et al. Mediators of the association between depression and role functioning. Acta Psychiatr Scand 2008; 118: 451-458.
[45] Beblo T, Mensebach C. Neuropsychologie der Borderlin-Persönlichkeitsstörung. In: Lautenbacher S, Gauggel S, Hrsg. Neuropsychologie psychischer Störungen. Berlin Heidelberg: Springer; 2010: 383-398.

[46] Dinn WM, Harris CL, Aycicegi A et al. Neurocognitive function in borderline personality disorder. Prog Neuropsychopharmacol Biol Psychiatry 2004; 28: 329-341.

[47] Hengartner MP, Müller M, Rodgers S et al. Occupational functioning and work impairment in association with personality disorder trait-scores. Soc Psychiatry Psychiatr Epidemiol 2014; 49: 327-335.

[48] Zanarini MC, Jacoby RJ, Frankenburg FR et al. The 10-year course of social security disability income reported by patients with borderline personality disorder and axis II comparison subjects. J Pers Disord 2009; 23: 346-356.

[49] Juurlink TT, Have M ten, Lamers F et al. Borderline personality symptoms and work performance: a population-based survey. BMC Psychiatry 2018; 18: 202.

[50] Lautenbacher S. Neuropsychologie der Angststörungen. In: Lautenbacher S, Gauggel S, Hrsg. Neuropsychologie psychischer Störungen. Berlin Heidelberg: Springer; 2010: 189-198.

[51] Craske MG, Stein MB, Eley TC et al. Anxiety disorders. Nat Rev Dis Primers 2017; 3: Article 17024.

[52] Muschalla B. Work-anxiety rather than cognitive performance contributes to workability decisions in patients with mental disorders. J Occup Environ Med 2018; 60: 1042-1048.

[53] Handel M. Trends in Job Skill Demands in OECD Countries. OECD Social, Employment and Migration Working Papers 2012.

[54] Then FS, Luck T, Luppa M et al. Association between mental demands at work and cognitive functioning in the general population - results of the health study of the Leipzig research center for civilization diseases (LIFE). J Occup Med Toxicol 2014; 9: 23.

[55] Green MF, Llerena K, Kern RS. The "Right Stuff" revisited. What have we learned about the determinants of daily functioning in cchizophrenia? Schizophr Bull 2015; 41: 781-785.

[56] Nordahl H, Wells A. Social anxiety and work status: the role of negative metacognitive beliefs, symptom severity and cognitive-behavioural factors. J Ment Health 2017: 1-5.

[57] Donohoe G, Schmidt H, Robertson IH. Cognitive remediation therapy for schizophrenia: what is it and does it work? Ir J Psychol Med 2011; 28: 217-221.

[58] Greig TC, Zito W, Wexler BE et al. Improved cognitive function in schizophrenia after one year of cognitive training and vocational services. Schizophr Res 2007; 96: 156-161.

[59] van Duin $D$, Winter $L$ de, Oud $M$ et al. The effect of rehabilitation combined with cognitive remediation on functioning in persons with severe mental illness: systematic review and meta-analysis. Psychol Med 2019: 1-12.

[60] Hoertnagl CM, Hofer A. Social cognition in serious mental illness. Curr Opin Psychiatry 2014; 27: 197-202.

Bibliografie

DOI https://doi.org/10.1055/a-0888-4926

Nervenheilkunde 2019; 38: 474-478

(c) Georg Thieme Verlag KG Stuttgart · New York ISSN 0722-1541 


\section{Punkte sammeln auf CM/F.thieme.de}

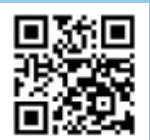

Diese Fortbildungseinheit ist in der Regel 12 Monate online für die Teilnahme verfügbar. Den genauen Einsendeschluss finden Sie unter https://eref.thieme.de/CXCX3YG.

Sollten Sie Fragen zur Online-Teilnahme haben, finden Sie unter https://cme.thieme.de/hilfe eine ausführliche Anleitung. Wir wünschen viel Erfolg beim Beantworten der Fragen!

Unter https://eref.thieme.de/CXCX3YG oder über den QR-Code kommen Sie direkt zur Startseite des Wissenstests.

VNR 2760512019156645366

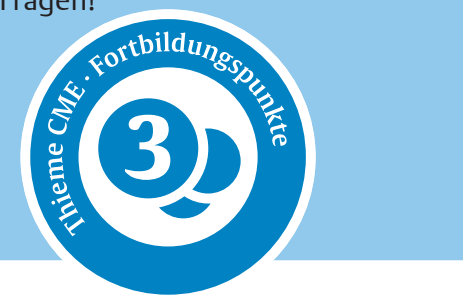

\section{Frage 1}

Welche der folgenden kognitiven Leistungsdomänen erwiesen sich in der Prognose funktionalen Outcomes bei Schizophrenie als am wenigsten relevant?
A exekutive Kontrollfunktionen
B sprachliche Fähigkeiten
C Verarbeitungsgeschwindigkeit
D Arbeitsgedächtnis
E Konzentrationsfähigkeit

\section{Frage 2}

Neuere berufliche Rehabilitationsprogramme versuchen, Patienten mit schweren psychischen Störungen rasch in eine sozialversicherungspflichtige Beschäftigung zu integrieren und dabei zu unterstützen, diese Anstellung zu halten. Welche der folgenden Formulierungen entspricht am wenigsten diesen neuen Konzepten?

A Individual Placement an Support

B Supported Employment

C Rehabilitation nach dem Prinzip des Place-and-Train

D Rehabilitation nach dem Prinzip „erst Trainieren, dann Platzieren“

E Rehabilitation nach dem Prinzip der unterstützten Beschäftigung

\section{Frage 3}

Welche der folgenden Aussagen zu kognitiven Beeinträchtigungen bei Schizophrenie ist richtig? Kognitive Beeinträchtigungen bei Schizophrenie sind ...

A in Qualität und Ausmaß eng an die Akuität positiver Symptome gekoppelt und in symptomatischer Remission nicht nachweisbar.

B zeitlich maximal instabil und prognostisch nicht valide.

$C$ in der Regel erst nach mehreren Erkrankungsepisoden feststellbar.

D Folge neuroleptischer Langzeittherapie.

E sowohl während als auch nach Abklingen florider Symptome festzustellen.

\section{Frage 4}

Kognitive Beeinträchtigungen bei bipolar affektiven Störungen ...

A schließen in der Regel Störungen der exekutiven Kontrollfunktionen ein.

B sind in der Regel stärker ausgeprägt als bei Patienten mit Schizophrenie.

C betreffen ein spezifisches Minderleistungsprofil aus Orientierungsstörung und reduzierter Fehlerzahl.

D persistieren nicht in euthyme Erkrankungsphasen.

E haben keine prognostische Relevanz.

\section{Frage 5}

Welche Aussage zu kognitiven Beeinträchtigungen von Patienten mit unipolar affektiven Erkrankungen ist am ehesten richtig?

A Kognitive Störungen bei Depression sind in der Regel stärker ausgeprägt als bei Schizophrenie.

B Kognitive Störungen sind ausführlich evaluierte Prognosefaktoren beruflicher Wiedereingliederung bei Depression.

C Kognitive Störungen bei Depression ähneln qualitativ, nicht aber quantitativ den Beeinträchtigungen bei Schizophrenie.

D Exekutive Kontrollfunktionen sind in der Regel nicht beeinträchtigt.

E Aufmerksamkeitsfunktionen sind in der Regel nicht beeinträchtigt.

\section{Frage 6}

Patienten mit schweren Persönlichkeitsstörungen ...

A weisen immer auch schwere Aufmerksamkeitsstörungen auf.

B weisen immer auch schwere Gedächtnisstörungen auf.

C weisen in keinem Fall Störungen der Exekutivfunktionen auf.

D weisen zeitlich variable und vergleichsweise geringgradige kognitive Störungen auf.

E sind in Programmen beruflicher Rehabilitation zunehmend seltener anzutreffen. 


\section{Punkte sammeln auf CME.thieme.de}

Fortsetzung ...

\section{Frage 7}

Für Patienten mit Angststörungen gilt am ehesten, dass ...

A sie nicht in Programme des Supported Employment integriert werden können, da zunächst ein Kompensationstraining kognitiver Störungen erfolgen muss.

B sich kognitive Defizite in mehreren Metaanalysen als valide Prädiktoren einer erfolgreichen Wiedereingliederung erwiesen haben.

C sich deren berufliche Perspektive weniger durch kognitive Leistungen als vielmehr durch das Ausmaß berufsbezogener Ängste vorhersagen lässt.

D sich durch angstassoziierte Aktivität im limbischen System die Encodierung bedrohlicher Reize maximal abschwächt.

E durch Hypofrontalität exekutive Funktionen verstärkt sind.

\section{Frage 8}

Programme der Rehabilitation sollten zur Berücksichtigung kognitiver Beeinträchtigungen psychisch Kranker ...

A von der Vermittlung in Arbeitsstellen des ersten Arbeitsmarktes grundsätzlich absehen, um neuerliches Scheitern der Betroffenen zu verhindern.

B kognitive Leistungsstörungen diagnostizieren und ggf. durch kognitive Trainings zu kompensieren versuchen.

C keine besonderen diagnostischen Maßnahmen und Hilfen beinhalten.

D mit von Defiziten betroffenen Teilnehmern primär eine EU-Berentung anstreben.

E im Sinne von fördern und fordern in Arbeitsstellen integrieren, die über den kognitiven Fähigkeiten der Betroffenen angesiedelt sind.

\section{Frage 9}

Welches der folgenden Merkmale ist zur Prognose beruflicher Wiedereingliederung psychisch Kranker im Vergleich am wenigsten geeignet?
A Kognitive Fertigkeiten
B Metakognition zum Thema Arbeit und Beschäftigung
C Soziale Kognition
D die bisherige berufliche Entwicklung
E Positivsymptomatik bei Erkrankungsbeginn

\section{Frage 10}

Welche der folgenden Entwicklungen zeigen sich in Bezug auf berufliche Rehabilitationsmaßnahmen am wenigsten?

A Zunahme des relativen Anteils von Patienten mit psychotischen Störungen

B Zunehmend mehr Programme des Place-and-Train-Ansatzes

C Zunehmend stärkere Integration kognitiver Trainings in die berufliche Rehabilitation

D Erhöhung von AU-Tagen wegen psychischer Erkrankungen in der Allgemeinbevölkerung

E Zunehmend breiteres Spektrum psychischer Erkrankungen in der Klientel beruflicher Rehabilitation 